\title{
Pattern Classification of Partial Discharge Data
}

\author{
Sung-Ho Kim*, Geum-Dong Bae* \\ * School of Electronics and Information Engineering, College of Engineering \\ Kunsan National University
}

\begin{abstract}
$\mathrm{PD}$ (Partial discharges) are small electrical sparks that occur within the electric insulation of cables, transformers and windings on motors. PD analysis is a proactive diagnostic approach that uses PD measurements to evaluate the integrity of this equipment. Recently, several diagnostic algorithms for classifying the type of PD and locating the defect position have been developed. In this work, a new PD recognition system is proposed, which utilizes approximate coefficients of wavelet transform as a feature vector, furthermore, introduces bank of Elman networks to recognize the various PD phenomena. In order to verify the performance of the proposed scheme, it is applied to the simulated PD data.
\end{abstract}

Key Words : Discharge, Wavelet, Elman network

\section{Introduction}

Diagnostic techniques constitute a fundamental tool for an optimized management of power networks. An efficient monitoring and diagnostic system can reduce costs for maintenance and repair operations. It is consolidated knowledge that partial discharge(PD) measurements constitute one of the most promising tools for the evaluation of the insulation deterioration. PD has been known to be related to the degradation of insulation materials or the lifetime of high voltage power apparatus and cables. Recently, several diagnostic schemes for detecting the insulation deterioration of the power cables have been developed[1-2]. The main trend of these researches is to develope an algorithm for identifying the types of PD, locating the defect location and measuring the insulation deterioration of the power cables [3]. Some of these works utilize the 2- or 3-dimensional statistical discharge patterns, such as $\Phi-\mathrm{Q}-\mathrm{N}, \Phi-\mathrm{Q}$ and $\Phi-\mathrm{N}[4]$. But, the method that uses the statistical distribution of parameters needs a complex computation process, large number of input data, reacts to even small variations of the PE's values in classification of results, shows many changes, all these being weak points in this method. and The method that uses graph forms gets graph data and due to the fact that the characteristic feature of partial discharge is that it happens instantly, data accumulated in a predetermined time should be used. Consequently, the recognition of noise is not easy and also conducting the noise removal in data is not an easy process. On the other side, the researches that apply neural networks become actively accomplished due to the ability of fast computers. Basically thinking, if neural networks are trained with some electric equipment system scheme, the

Manuscript received Nov. 25, 2005; revised Dec. 8, 2005. This work has been supported by KESRI(R-2003-B-274), which is funded by MOCIE(Ministry of commerce, industry and energy). output value is not the value of the stationary state when the abnormality occurs but a different value. This time we can know that it is an abnormal condition. For neural networks there is a large number of algorithms, so, it is desirable to choose the appropriate algorithm for recognition of partial discharge in cables insulation.

As I mentioned before, a large amount of data is needed, but obtaining these test data is a very difficult process because of the characteristic feature of PD. Also, due to the inconsistence of the pattern recognition caused by noise, noise removal becomes a very important issue. In order to solve all these problems, we will use the wavelet method. However, a common drawback of these methods is the presence of substantial noise. In general, PD data are distorted by noise, but useful information can be extracted from the deformed data. Every PD pattern has its own unique shape, which forms the basis of PD recognition. The appropriate definition of feature is essential in recognition. PD signals with well-selected features mean high recognition rate.

Recently, wavelet transforms have recently emerged as powerful tools for a broad range of applications, such as signal compression. The wavelet transform has good localization in both frequency and time domains. In the fields of data compression, wavelet transform is used to exploit the redundancy in the signal. The performance of a wavelet transform for data compression lies in its ability in concentrating a large percentage of total signal energy in a few coefficients. After the original signal is transformed into the wavelet coefficients, many coefficients are so small that these coefficients can be omitted without losing significant information.

In this work, PD recognition system based on both wavelet and neural network is proposed. It uses wavelet coefficients as a feature vector and also utilizes Elman network to recognize the phenomena caused by various PD activities. Elman network plays an important role as one-step ahead predictor, which can estimate the predicted future value.

This paper is organized as follows: In section 2, 
characteristics of PD are briefly discussed. In section 3, PD recognition system using wavelet and Elman network is described. The performance of the proposed system is evaluated, and the results are discussed in section 4 and 5 .

\section{Characteristics of PD}

PD can be described as small electric sparks that occur within the electric insulation of cables, transformers and windings in motors owing to various voids[5]. This spark partially bridges the gap between phase insulation to ground, or phase to phase insulation. The voids may be located between the copper conductor and insulation wall, or internal to the insulation itself as in Fig.1. The discharges are small arcs occurring within the insulation system, therefore it can deteriorate the insulation, and can result in eventual complete insulation failure.

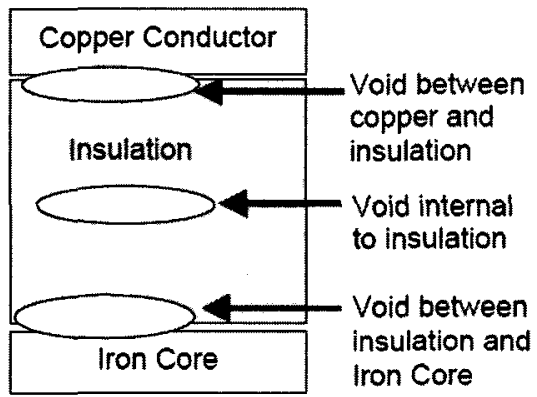

Fig. 1. Voids within the insulation system

The characteristic of PD is that it occurs only during the first and third quarter of each cycle. Effectively, during the initial rising positive signal, all of the capacitive components are being charged until the partial discharge inception voltage is reached across each specific void, and partial discharge commence. When the positive cycle begins to decrease, the positive voltage across each void is reduced. During the third quarter cycle, this positive charge is effectively reverse, resulting in a positive charge in the reverse direction as in Fig. 2.

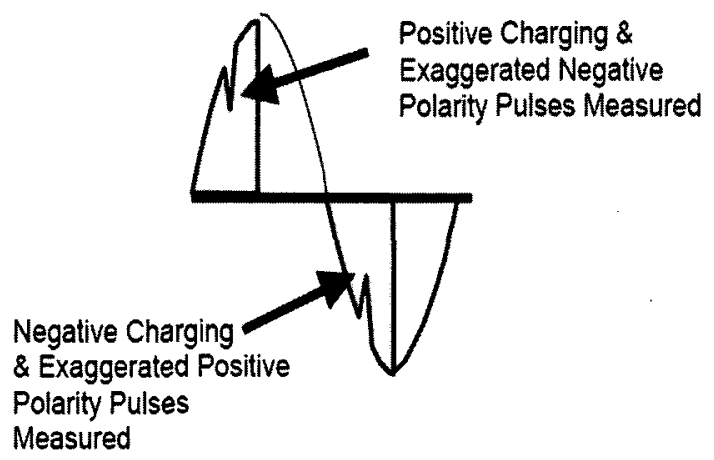

Fig. 2. PD occurrence during each cycle
Actual PD events can be measured by using digital storage oscilloscope. PD events are observed by recording the current induced in the high voltage electrode. The peak values of the current and its time relative to the phase of the applied voltage are accumulated into the internal memory of digital oscilloscope as in fig. 3.

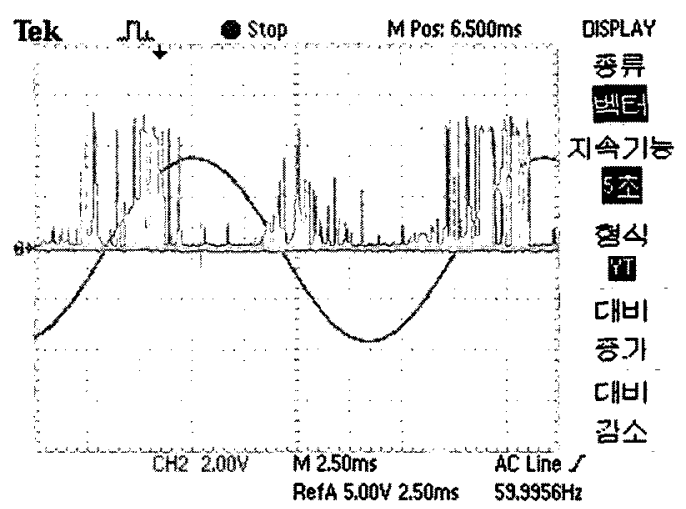

(a) PD occurrence 1

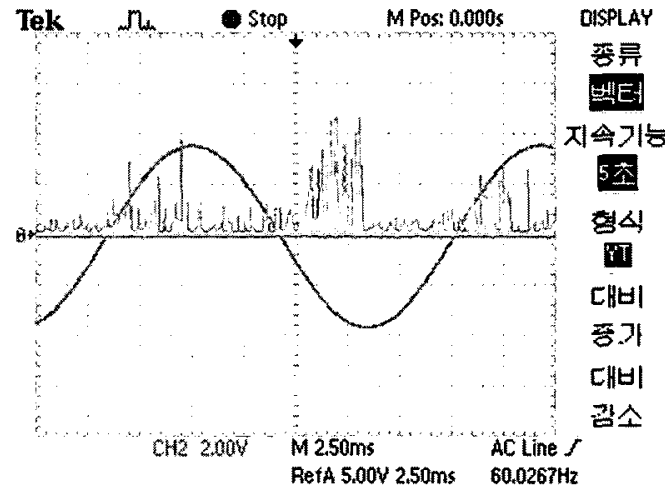

(b) PD occurrence 2

Fig. 3. Phase-magnitude plots for different PD types

\section{PD recognition system based on wavelet and neural network}

The proposed PD recognition system based on wavelet and neural network is shown in fig.4.

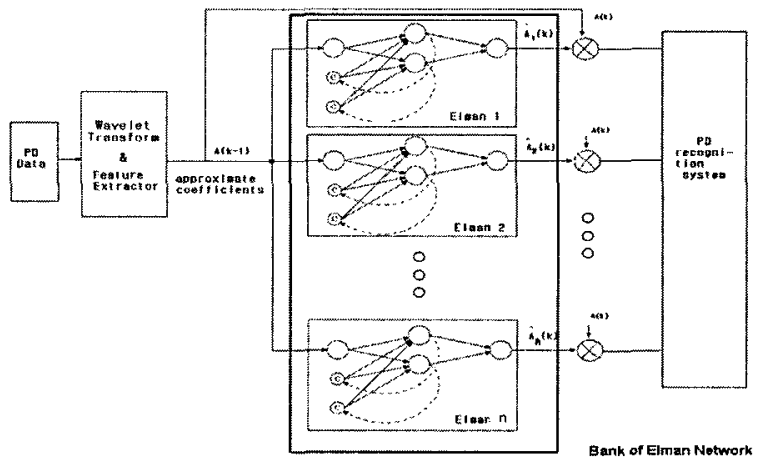

Fig. 4. The structure of the proposed PD recognition system 
In fig. 4, input represents the approximate coefficients, which can be thought of a sequence. Sequence of approximate coefficients is presented to each Elman network.

\subsection{Wavelet and feature extraction}

Wavelet transforms have recently emerged as powerful tools for a broad range of applications, such as signal compression[6-8]. It has good localization in both frequency and time domains, having fine frequency resolution and coarse time resolution and fine time resolution at lower frequency. In the fields of data compression, it is used to exploit the redundancy in the signal. The performance of a wavelet transform for data compression lies in its ability in concentrating a large percentage of total signal energy in a few coefficients. After the original signal is transformed into the wavelet coefficients, many coefficients are so small that these coefficients can be omitted without losing significant information. The multi-resolution signal analysis used in this work is briefly introduced.

Multi-resolution signal analysis decomposes a function into a smooth approximation of the original function and a set of detailed information at different resolutions. Formally, let $L^{2}(R)$ denote all functions with finite energy; the smooth approximation of a function $f \in L^{2}(R)$ at any resolution $2^{i}$ is a projection denoted as $A_{2}: L^{2}(R) \rightarrow V_{2^{\prime}}$, $V_{2^{i}} \in L^{2}(R)$, and the detail of $f$ at any higher resolution $2^{j}$ is a projection of $f$ onto a subspace. $O_{2}$; of $L^{2}(R)$ denoted as $P_{2} ; L^{2}(R) \rightarrow O_{2} ;, j \geq i$. Consequently, the finest detailed information is contained in $P_{2}$, with the highest resolution. For the discrete functions, it can be proven that there exist two families of functions:

$$
\begin{aligned}
& \phi_{j, n}=2^{-j / 2} \phi\left(2^{j} t-n\right)_{n \in Z} \\
& \phi_{j, n}=2^{-j / 2} \phi\left(2^{j} t-n\right)_{n \in Z}
\end{aligned}
$$

which constitute the ortho-normal basis of $\mathrm{V}_{2}$; and $\mathrm{O}_{2}$, respectively. $\psi_{j, n}$ are called wavelets and $\Phi_{j, n}$ are the corresponding scaling functions.

Using wavelets and scaling functions, the discrete detail signal and discrete approximation at resolution $2^{j}$ are respectively defined as:

$$
\begin{aligned}
& \left(D_{2^{j}} f\right)_{n}=2^{-j / 2}\left\langle f(u), \phi_{j, n}\right\rangle \\
& \left(A_{2^{d}}^{d} f\right)_{n}=2^{-j / 2}\left\langle f(u), \phi_{j, n}\right\rangle
\end{aligned}
$$

Instead of calculating the inner products in eq.(3),(4), a pyramidal algorithm is applied for the decomposition of the function, and the detailed process is shown in fig. 5 .

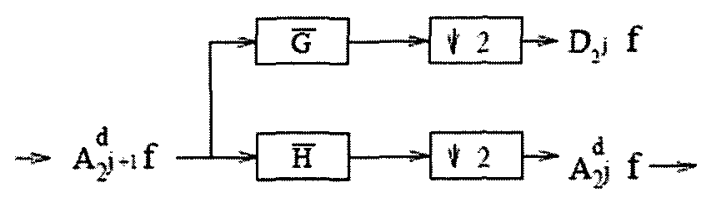

Fig. 5. Wavelet decomposition process
In this work, the wavelet coefficients which can be thought of as a sequence are utilized as a feature vector for the proposed PD recognition system. Although there are many good alternatives for selecting the feature vectors, it's not sensible to use too many of them. If the dimension of these feature vector is big, it is difficult to directly use them as training data set for neural classifier. Generally, up to ten features is a resonable choice. Therefore, it is required to utilize a certain method for dimension reduction of feature vectors.

\subsection{Bank of Elman network and PD recognition}

Elman network may be described in analogy to three layer feedforward network, except it has a feedback connection from output of hidden layer to input layer[9-11]. The output of the hidden units at step k-1 are copied to context units and used together with the new inputs at step $k$ to yield the next hidden units output, as shown in fig. 6 .

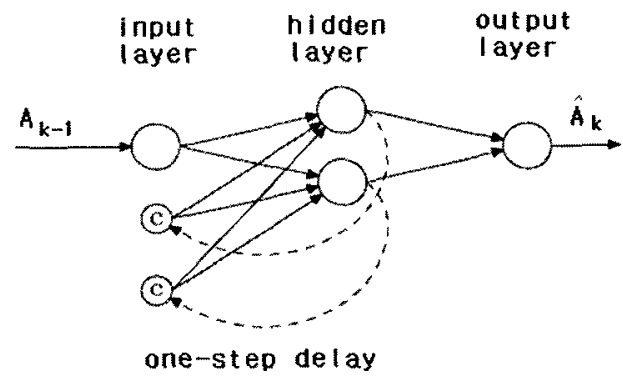

Fig. 6. "Structure of Elman network

If Elman network is trained to memorize the wavelet coefficient sequence related with a certain PD pattern, the trained Elman network can be effectively used as a predictor for predicting successive elements of that sequence. Each trained Elman networks for corresponding PD pattern sequences can be structured as a bank. Therefore, if unknown wavelet coefficient sequence is presented to each Elman networks as shown in fig. 4, residuals between the predicted coefficient value and the real one can be calculated. These residuals are further utilized to recognize which class the current PD pattern belongs to.

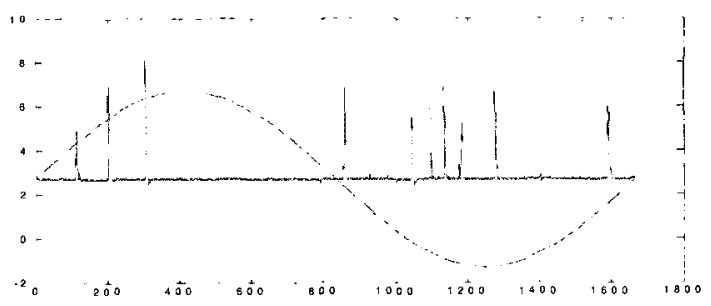

(a) type $\mathrm{A}$ 


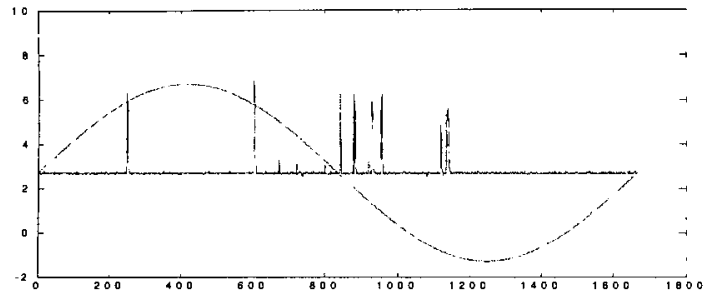

(b) type $B$

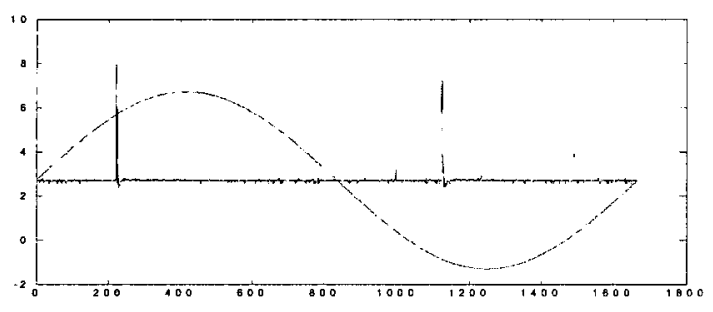

(c) type $\mathrm{C}$

Fig. 7. Simulated PD patterns

\section{Application of the proposed scheme to PD pattern}

In order to verify the performance of the proposed scheme, it is applied to the simulated PD patterns. The measurement of accumulated partial discharge according to the phase is shown in fig. 7.

Extracting feature vector from PD patterns shown in fig. 7 is one of the most important steps in pattern recognition. For this, wavelet transform is applied to these patterns. Approximate coefficient corresponding to each PD pattern can be obtained as a result of wavelet transform. These coefficient can be thought of as a feature vector.

Each Elman network which acts as one-step ahead predictor should be trained to memorize each sequence shown in fig. 8 . If unknown coefficient sequence corresponding to a certain PD pattern is presented to each Elman networks, residuals between the predicted coefficient value and the real one can be calculated at each step. The sum of residual at each step is shown in fig. 9.

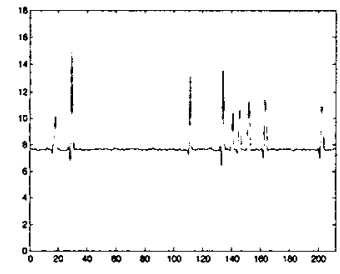

Approximate coefficients in case of level depth 3 in wavelet transform

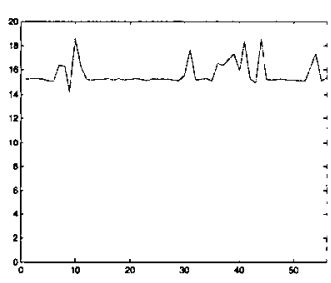

Approximate coefficients in case of level depth 5 in wavelet transform

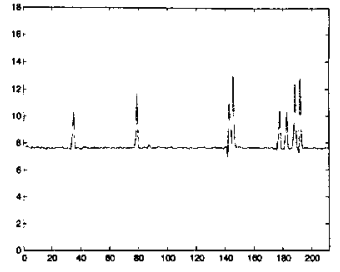

Approximate coefficients in case of level depth 3 in wavelet transform (b) type B

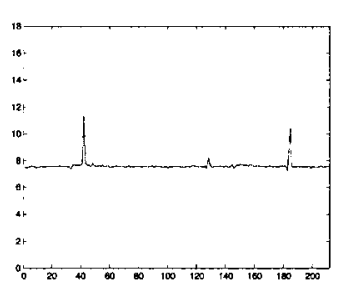

Approximate coefficients in case of level depth 3

in wavelet transform

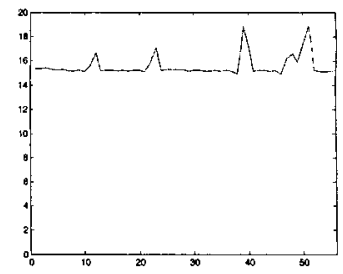

Approximate coefficients in case of level depth 5 in wavelet transform

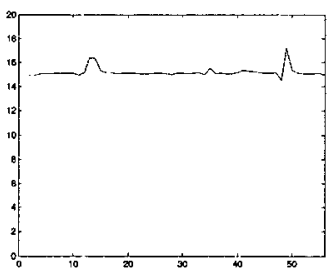

Approximate coefficients in case of level depth 5 in wavelet transform

(c) type $\mathrm{C}$

Fig. 8. Approximate coefficients from wavelet transform corresponding to each $\mathrm{PD}$ pattern

Table 1. Sum Square Error(SSE) of residual between the original and predicted approximate coefficient

\begin{tabular}{|c|c|c|c|c|}
\hline \multirow{2}{*}{ level-depth } & \multicolumn{2}{|c|}{ Sum Square Error of residual } & \multirow{2}{*}{$\begin{array}{c}\text { Recognition } \\
\text { Result }\end{array}$} \\
\cline { 2 - 4 } & elman1 & elman2 & elman3 & type A \\
\hline 3 & 0.0139 & 0.0152 & 0.1356 & type A \\
\hline 5 & 0.0013 & 0.0019 & 0.0059 & typen \\
\hline
\end{tabular}

Table 2. Recognition ratio according to level-depth in wavelet transform

\begin{tabular}{|c|c|c|c|c|c|}
\hline \multirow{2}{*}{$\begin{array}{c}\text { level- } \\
\text { depth }\end{array}$} & \multirow{2}{*}{$\begin{array}{c}\text { pattern } \\
\text { type }\end{array}$} & \multicolumn{2}{|l|}{ Sum Square Error of residual } & Recognition \\
\cline { 3 - 6 } & elman1 & elman2 & elman3 & Result \\
\hline \multirow{3}{*}{3} & type A & 0.0199 & 0.0245 & 0.1077 & type A \\
\cline { 2 - 6 } & type B & 0.0270 & 0.0101 & 0.0145 & type B \\
\cline { 2 - 6 } & type C & 0.0083 & 0.0080 & 0.0036 & type C \\
\hline \multirow{3}{*}{5} & type A & 0.0137 & 0.0098 & 0.0114 & type B \\
\cline { 2 - 6 } & type B & 0.0089 & 0.0334 & 0.0112 & type A \\
\cline { 2 - 6 } & type C & 0.0032 & 0.0366 & 0.0065 & type A \\
\hline
\end{tabular}

(a) type $\mathrm{A}$ 


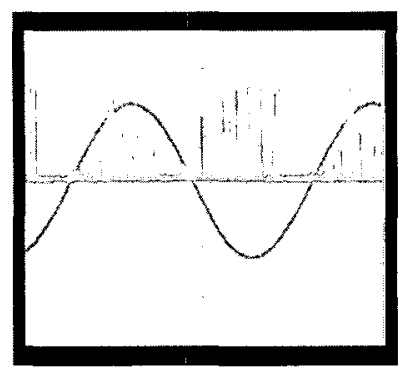

(a) Inputted PD Pattern (type-A)

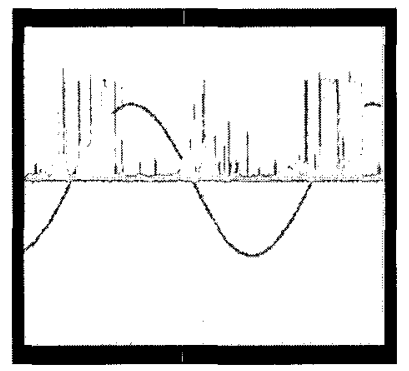

(a) Inputted PD Pattern (type-B)

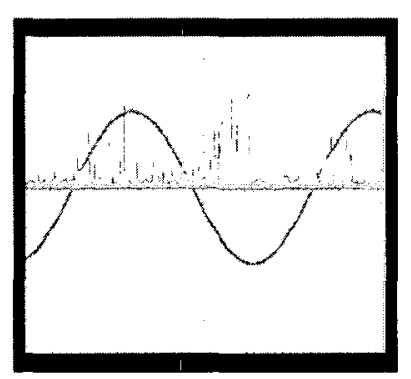

(a) Inputted PD Pattern (type-C)

Fig. 9. Trend of Sum Square Error when the decomposition level-depth is 3

As you can see, the proposed recognition system shows a desirable recognition performance. The recognition ratio varies depending on the decomposition level-depth in wavelet transform as in table 1. Table 2 shows that selection of appropriate level-depth is required to get a good diagnostic result.

\section{Conclusion}

In this work, PD recognition system which utilizes wavelet transform and Elman network is proposed. Wavelet coefficient which can be thought of as good feature vector plays a key role in the proposed system. The simulation result shows that level-depth for decomposition process in wavelet transform should be carefully selected to get a better recognition.
Furthermore, Elman network which acts as one-step ahead predictor is good for efficient PD recognition system. However, the proposed PD recognition system should be further investigated with many real PD pattern data to verify the practical applicability.

\section{REFERENCES}

[1] C. Chang and Q. Su, "Partial discharge measurement and analysis using an intergrated system", Center for Electrial Power Engineering, Monash University, Clayton VIC 3168 Austrailia

[2] M. de Nigris et al., "Cable diagnosis based on defect location and characterization through partial discharge measurements", http://www.cigre.org, CIGRE Session 2002, 15-109, 2002

[3] Park, Sung Hee, "Discrimination of Complex Partial Discharge Sources by Neural Networks", Dept. Electrical Engineering, Graduate School, Chunbuk National University, Cheonju, Korea, 2004

[4] R.Bartnikas, "Partial Discharges . Their mechanism. Detection and measurement", IEEE,Trans.on EL. Vol.9. No. 5, p.763-778, 2002

[5] G. Paoletti and A. Golubev, "Partial Discharge Theory and Applications to Electrical Systems", the 1999 IEEE IAS Pulp and Paper Industry Conference, Seattle, 1999

[6] wavelet algorithm Wavelet Toolbox: Advanced Concepts: Algorithms, software "matlab6.5" help document

[7] Brani Vidakovic, "Statistical Modeling by Wavelets", Wileyseries in probability and statistics, 1999

[8] Howard L. Resnikoff and Ramond O. wells, Jr. "Wavelet analysis' The scalable structure of information"', Springer, 1998

[9] elman network http://www.cs.wright.edu/ mrizki /cs765/ Lectures/Lecture12.ppt\#315,24, Elman Algorithm

[10] T. Lane and C.E. Brodley, "An application of machine learning to anomaly detection.", In Proceedings of the 20th National Information Systems Security Conference, p.366\{377, October 1997. 1998 IEEE Computer Society, IEEE Computer Society Press. Scottsdale, AZ.

[11] T. J. Cholewo and J. M. Zurada, "Sequential Network Construction for Time Series Prediction", IEEE international joint Conference on Neural Networks, p.2034-2039, Houston, Texas, USA, June 9-12, 1997

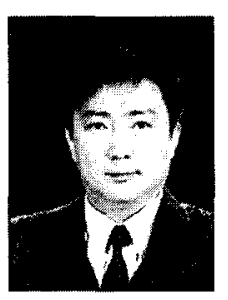

Sung-Ho Kim

Sung-Ho Kim received B.S., M.S and Ph.D. degrees in electrical engineering from Korea University, Seoul, Korea, in 1984, 1986 and 1991 respectively, currently, he is a professor of the school of electronics and information engineering, Kunsan National University, Kunsan, Korea. His current research and teaching activities are in the area of intelligent control and web-based remote monitoring and fault diagnostic system using fuzzy logic and neural network. 
International Journal of Fuzzy Logic and Intelligent Systems, vol. 5, no. 4, December 2005

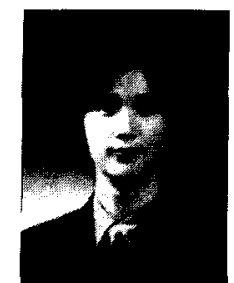

\section{Geum-Dong Bae}

Geum-Dong Bae received B.S. degree in the school of electronics and information engineering, Kunsan National University, Kunsan, Korea in 2004. He is pursuing M.S degree from the school of electronics and information engineering, Kunsan National University, Kunsan, Korea. His research interests web-based remote monitoring system and fault diagnostic system using fuzzy logic and neural network 